\title{
Gene- and Viral-Based Therapies for Brain Tumors
}

\author{
Kaveh Asadi-Moghaddam and E. Antonio Chiocca \\ Department of Neurological Surgery, Dardinger Center for Neuro-oncology and Neurosciences, James Cancer Hospital/Solove \\ Research Institute, The Ohio State University Medical Center, Columbus, Ohio 43210-1240
}

\begin{abstract}
Summary: Advances in understanding and controlling genes and their expression have set the stage to alter genetic material to fight or prevent disease with brain tumors being among one of the first human malignancies to be targeted by gene therapy. All proteins are coded for by DNA and most neoplastic diseases ultimately result from the expression or lack thereof with one or more proteins (e.g., coded by oncogenes or tumor suppressor genes, respectively). In theory, therefore, diseases could be treated by expression of the appropriate protein in the affected cells. Gene therapy is an experimental treatment that involves introducing genetic material (DNA or RNA) into cells, and it has made important advances in the past decade. Within this short time span, it has moved from the conceptual labora-
\end{abstract}

tory research stage to clinical translational trials for brain tumors. The most efficient approaches for gene delivery are based on viral vectors, which have been proven relatively safe in the $\mathrm{CNS}$, despite occasional cases of morbidity and death in nonneurosurgical trials. However, the human response to various viral vectors can not be predicted in a reliable manner from animal experimentation, nor can size, consistency, and extent of experimental brain tumors in mouse models reflect the large, necrotic, infiltrative nature of malignant gliomas. Furthermore, the problem of delivering genetic vectors into solid brain tumors and the efficiency in situ gene transfer remains one of the most significant hurdles in gene therapy. Key Words: Glioma, gene therapy, oncolytic virus, clinical trial.

\section{INTRODUCTION}

Two crucial considerations in gene therapy relate to: 1) what gene should be delivered for expression, and 2) how to deliver it. In its simplest form, gene therapy is the process by which either defective or missing genes are replaced or new functions to the host's cells are introduced. For this purpose, the genetic material is coupled to additional regulatory sequences (e.g., promoters and enhancers) and is packaged inside a gene delivery vehicle to enable transfer and expression of the intended gene product inside the cell (FIG. 1).

Although conceptually straightforward, the efficient expression of foreign genes is the most critical aspect for the success of in vivo gene therapy. The first step of gene therapy involves gene delivery to facilitate the expression of the therapeutic gene in the interior of a cell. The simplest method is the direct introduction of therapeutic DNA into target cells by physical (i.e., electroporation) or chemical techniques (i.e., lipofection). ${ }^{1}$ This approach still remains limited in its application, because it is relatively inefficient,

Address correspondence to: E. Antonio Chiocca, M.D., Ph.D., Department of Neurological Surgery, Dardinger Center for Neuro-oncology and Neurosciences, N-1017 Doan Hall, 410 W. 10th Avenue, James Cancer Hospital/Solove Research Institute, The Ohio State University Medical Center, Columbus, OH 43210-1240. E-mail: ea.chiocca@osumc.edu. it can be used only with certain tissues and requires large amounts of DNA. Furthermore, among the several barriers to successful gene delivery, foreign genes and/or the vectors used to deliver them can trigger a range of immune responses. However, sometimes, these immune responses have been harnessed behind the concept of using gene therapy as a vaccine (i.e., DNA vaccines). ${ }^{2}$

The next difficulty for the foreign genetic material is that once within the cell, it must escape intracellular degradation to enter the nucleus to be expressed (FIG. 1). ${ }^{3,4}$ Therefore, gene delivery systems (vectors) were designed to protect the genetic material. An ideal vector needs to meet three criteria: 1) it should protect the transgene against degradation by nucleases in the extracellular matrix, 2) it should bring the transgene across the plasma membrane and into the nucleus of target cells, and 3) it should have no detrimental effects. Currently, such vectors of gene transfer can be classified into two categories: 1) viral and 2) nonviral.,

\section{GENE THERAPY APPROACHES FOR GLIOMAS: FIVE TYPES OF DELIVERY}

Five glioma gene therapy approaches are currently being explored: 1) pro-drug activating gene therapy (also 


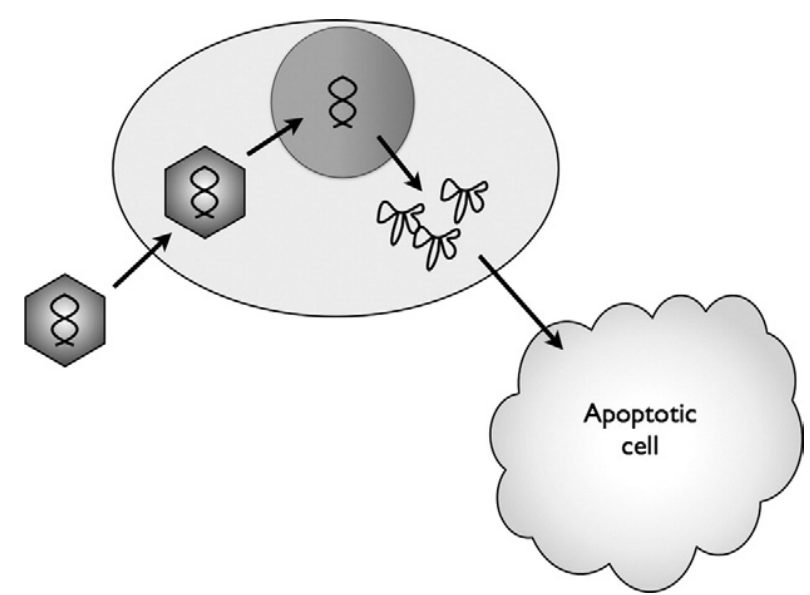

FIG. 1. Mechanism of vector-mediated gene therapy. A viralbased vector binds to the cell membrane, which elicits entry into the cell. The viral DNA then travels to the nucleus, where it becomes active. In the nucleus, viral DNA becomes extrachromosomal and gene expression ensues. As the gene becomes transcribed into mRNA and then mRNA becomes protein, the desired therapeutic effect occurs. In this case, the desired therapeutic effect (e.g., cell death of the tumor cell) was to cause apoptosis by introduction of a pro-apoptotic gene, as an example.

called suicide gene therapy, chemotherapy-sensitizing gene therapy, gene-directed enzyme pro-drug therapy); 2) transfer of tumor suppressor genes and cell cycle modulators; 3) genetic immune modulation; 4) anti-angiogenic gene therapy; and 5) oncolytic viruses (OVs).

\section{Pro-drug activating}

The pro-drug-activating approach is the most commonly used technique in clinical trials for brain tumors. It involves transducing tumor cells with a gene encoding an enzyme that can metabolize a nontoxic pro-drug to its toxic form.

Herpes simplex virus type 1 (HSV-1) thymidine kinase. The HSV-1 thymidine kinase (HSVtk) is an enzyme that metabolizes nontoxic nucleoside analogs, such as ganciclovir (GCV), acyclovir, or valacyclovir (VCV), into a cytotoxic molecule. The GCV metabolite is incorporated into DNA, causing DNA elongation to terminate and subsequently cause cell death. ${ }^{7}$ Because the effects of GCV is limited to DNA, it primarily targets the mitotic cells, much like the $\mathrm{S}$ phase-specific chemotherapeutic agents, but it also affects the ability of DNA to be repaired by DNA polymerases, and thus it theoretically could also affect quiescent cells with damaged DNA. In vivo efficacy was demonstrated in multiple animal studies. $^{8-10}$ Furthermore, preclinical experiments demonstrated marked tumor elimination, despite gene transfer into only a small fraction of tumor cells. ${ }^{11}$ This cytotoxic effect of transduced cells on adjacent nontransduced cells is termed the bystander effect. ${ }^{12}$ The bystander effect is mediated mainly by the transfer of toxic phosphorylated forms of GCV to nontransduced cells, presumably via gap junctions. ${ }^{13}$ Another presumed mechanism contributing to the bystander effect is the targeting of mitotically active endothelial cells in tumor vessels. ${ }^{14}$ An immune-associated response against a nonhuman protein, such as HSVtk, leading to diffuse cell death that affects neighboring nontransduced cells has also been suggested. ${ }^{15}$ In addition to the bystander effect, tumor cells transduced to express HSVtk and treated with the antiviral agent acyclovir display enhanced sensitivity to radiation in culture and in vivo. ${ }^{16}$ Possible explanations for radiation enhancement could be that DNA that has incorporated acyclovir may be susceptible to radiation-induced strand breakage, and/or acyclovir might sensitize cells by inhibiting polymerase activity required for the repair of radiation-induced DNA damage. ${ }^{17}$ The HSVtk gene therapy approach was tested in human glioma trials (phase III) using RV vectors, and more recently adenovirus (AV) vectors. In fact, a phase III trial in Europe and Israel was recently completed under sponsorship by a British biotechnology venture (Ark Therapeutics) in which an AVHSV tk vector and GCV were provided to patients with newly diagnosed glioblastomas (GBMs) before application of standard therapy (radiation and/or temozolomide). Interim results from this trial are available on the company's website at www.arktherapeutics.com/main/products. php?content $=$ products_cerepro. To take advantage of the possible synergy between radiation-induced damage of DNA and the inability of DNA with incorporated VCV metabolites to repair itself, we have recently completed a phase I clinical trial for patients with newly diagnosed malignant gliomas, in which AV-HSVtk and VCV is used in conjunction with radiation (Chiocca et al., unpublished).

Cytosine deaminase/5-Fluorocytosine. 5-Fluorocytosine (5-FC) is an agent used to treat fungal infections (such as Candida albicans). 5-FC is a pro-drug converted into the active agent 5 -fluorouracil (5-FU) by cytosine deaminase (CD), which is uniquely expressed in certain fungi and bacteria. While 5-FC is nontoxic to human cells because of the lack of $\mathrm{CD}, 5-\mathrm{FU}$ is used to treat cancers like colon, pancreatic, and breast cancer. The toxic effects of 5-FU are mediated by its intracellular metabolites, which cause DNA strand breakage leading to cell death. ${ }^{18}$ Two preclinical studies for glioblastomas using an adenoviral vector carrying the CD gene demonstrated promising results. ${ }^{19}$ However, no clinical trial for brain tumors has used this gene transfer strategy so far.

Cytochrome $\mathbf{P 4 5 0}$ 2B1/cyclophosphamide. Cyclophosphamide (CPA) is a pro-drug that is activated by liver-specific enzymes of the cytochrome P450 family. The active form of CPA (i.e., phosphoramide mustard) is an alkylating agent that generates DNA cross links and consecutive DNA strand breaks. The efficacy of CPA in treating brain tumors has been limited by the fact that (although CPA crosses the blood-brain barrier) its active metabolites are poorly transported across the blood-brain barrier. $^{20}$ The rat cytochrome P450 2B1 (CYP2B1) activates CPA with high efficiency, ${ }^{21}$ and gene therapy 
using CYP2B1 to activate CPA was designed primarily for use in brain tumors, because other malignancies have ready access to the active metabolites of CPAs. The implantation of CYP2B1 expressing RV vectors was shown to induce regression gliomas in animal studies. ${ }^{22,23}$

Replacement of parts of the HSV-1 genome with the CYP2B1 gene has led to the design of an HSV-1 vector (rRp450) that can kill tumor cells through three modes: 1) using viral oncolysis, 2) rendering the infected cell sensitive to CPA, and 3) rendering the infected cell sensitive to GCV. Animal studies using subcutaneous tumors established from glioma cell lines in immunodeficient mice showed tumor regression only when the animals were treated with $\mathrm{rRp} 450, \mathrm{CPA}$, and GCV. ${ }^{18}$ Currently, no P450/CPA clinical trials for brain tumors have been conducted.

\section{Transfer of tumor suppressor genes and cell-cycle modulators}

Advances in understanding the underlying molecular and genetic mechanisms of gliomas provide a rational framework for the development of new treatments. ${ }^{24}$ The p53 tumor suppressor protein regulates cell-cycle progression and apoptosis in response to many external insults (e.g., DNA damage and oncogenic mutations). ${ }^{25}$ Mutation in the p53 gene (TP53) resulting in loss of its function are common in astrocytomas, and are also associated with tumor progression from low-grade astrocytoma to glioblastoma. ${ }^{26}$ Accordingly, the TP53 gene became an attractive candidate for gene transfer in an attempt to restore cell cycle regulation in TP53-mutated cells and induce apoptosis, even in tumors with intact functional genes, by causing enhanced expression of the gene product. ${ }^{27,28}$ Another commonly affected cell-cycle pathway in gliomas is the retinoblastoma protein/cyclindependent kinases/cyclin-dependent kinase inhibitors (CDKN) circuit. ${ }^{29,30}$ Preliminary studies restoring the genomic region of CDKN2A/CDKN2B in glioblastoma cell lines demonstrated tumor growth arrest or apoptosis. ${ }^{31}$ Another candidate for a gene therapy approach is the epidermal growth-factor receptor gene, which shows frequent amplification in primary GBMs. ${ }^{32}$ A clinical trial using an adenoviral vector to deliver the p53 gene in patients with recurrent GBMs has been conducted. ${ }^{33}$

\section{Genetic immune modulation}

Gene therapy approaches using genetic immune modulation enhance the immune response against tumors by expressing cytokines and lymphokines. The frequently used cytokines to achieve genetic immune modulation of tumors are interleukin (IL)- $2,{ }^{34,35}$ IL-4, ${ }^{36}$ interleukin$12,{ }^{37}$ interferon (IF)- $\beta,{ }^{38}$ IF- $\gamma,{ }^{39}$ and granulocyte-macrophage colony stimulating factor. ${ }^{40}$ Several studies have been performed by infecting tumor cells ex vivo with cytokine genes, arresting cell growth by irradiation, and then reimplanting the cells to sustain paracrine secretion of cytokines within the tumor. ${ }^{34,41}$ Another model introduces RV producer cells carrying immune modulating genes into the tumor so infection occurs in situ. ${ }^{36}$ Several phase I trials are currently underway using this strategy. However, for IL- 2 and IFN- $\gamma$ severe CNS toxicity has been reported when these cytokines were secreted by tumor cells intracranially. ${ }^{42}$ One clinical trial in humans with recurrent gliomas has been conducted where an AV vector was employed to deliver the gene for human IF- $\beta{ }^{43}$ Gene therapy can also be used to generate tumor vaccines by inducing tumor antigen presentation by antigen-presenting cells. Antigen-presenting cells (i.e., dendritic cells) can be harvested from peripheral blood or brain tumor biopsy specimens, which are transduced with the DNA, mRNA, and/or proteins coding for the tumor antigen(s), and then expanded in vitro before administration to the patient. Clinical trials using genebased approaches for GBM are currently in process.

\section{Anti-angiogenic gene therapy}

Neovascularization is a feature of malignant gliomas and is dependent on several potent angiogenic factors secreted by tumor cells. Vascular endothelial growth factor is an important overexpressed angiogenic factor in gliomas. It has been shown that in vivo transfer of a recombinant $\mathrm{AV}$ vector carrying the gene for vascular endothelial growth factor in an antisense orientation into gliomas inhibits tumor growth. ${ }^{44}$ Antisense oligonucleotides are single strands of DNA or RNA that can bind to a complementary RNA sequence. If binding takes place, this DNA/RNA hybrid can be degraded, subsequently preventing protein synthesis. Another study showed significant glioma inhibition by using a RV vector to deliver a signaling-defective vascular endothelial growth factor receptor, which forms inactive dimers with wild-type vascular endothelial growth factor receptor. ${ }^{45}$ In addition to being one of the gene replacement strategies described earlier, p53 might also have an anti-angiogenic effect due to the discovery that inducing wild-type $\mathrm{p} 53$ expression in astrocytomas causes release of an angiogenesis inhibitory factor. ${ }^{46}$ The intense neovascularization in malignant gliomas may enable a new intravascular modality of gene therapy for this disease. Although genetic vectors administered intravascularly are unlikely to penetrate the blood-brain barrier, intravascular delivery of vectors may target endothelial cells in the brain. So far, no trials have been conducted using this approach in gliomas.

\section{Oncolytic viruses and gene delivery vectors}

There are several methods for nonviral gene transfer. However, these methods have been relatively inefficient in their capacity to transfer genes to a sufficiently elevated number of cells, especially for in vivo gene delivery into brain tumors.

So far, the most effective way to transfer DNA into somatic cells remains the use of a viral-based delivery system. Vectors for brain tumor therapy can be divided 
into two categories: 1) replication-defective vectors, which we will name as a vector from here on, and 2) replication-competent (ie, replication-conditional and oncolytic viruses), which we will name as oncolytic viruses (OVs) from here on. In the first instance, the vector is derived from a virus from which all or most of the viral genes have been removed to minimize virus-mediated toxicity. In the second instance, selected viral genes are deleted or mutated so that viral targeting and/or replication can occur selectively in tumor versus endogenous neural cells. So far, the replication-defective vectors used in gene therapy trials of brain tumors have been based on retrovirus and $\mathrm{AV}$. In terms of replication-competent (oncolytic) viruses, the ones used in clinical trials of brain tumors have been HSV, AV, reovirus and Newcastle disease virus (Table 1). However, experimentally, almost any type of virus has been used either in a replication defective or replication-competent fashion.

Retroviruses. Retroviruses (RVs) are a class of enveloped viruses containing a single-stranded RNA mole-

Table 1. Comparison of Viral Vectors and OVs Used for Clinical Trials

\begin{tabular}{|c|c|c|}
\hline Vector or $\mathrm{OV}$ & Advantages & Disadvantages \\
\hline Retrovirus (Vector) & $\begin{array}{l}\text { - Low immunogenicity } \\
\text { - Persistent gene expression } \\
\text { - Specific for mitotic cells }\end{array}$ & $\begin{array}{l}\text { - Insertional mutagenesis } \\
\text { - Infects dividing cells only }\end{array}$ \\
\hline Adenovirus (Vector) & $\begin{array}{l}\text { - Infects dividing and nondividing cells } \\
\text { - Nonintegrating } \\
\text { - Widely available experience in its use, } \\
\text { production, and widely developed by } \\
\text { biotechnology industry }\end{array}$ & $\begin{array}{l}\text { - Short-term gene expression } \\
\text { - Very immunogenic }\end{array}$ \\
\hline Adenovirus (OV) & $\begin{array}{l}\text { - Safe in human brain } \\
\text { - Mutants that target p16/RB, p53 and other } \\
\text { tumor suppressor pathways have been } \\
\text { engineered } \\
\text { - Large experience in generation of clinical } \\
\text { product } \\
\text { - Most widely developed by biotechnology } \\
\text { industry } \\
\text { - One mutant has been approved for use in }\end{array}$ & $\begin{array}{l}\text { - Low transgene capacity } \\
\text { - Cell lysis is slow } \\
\text { - High number of viral particles } \\
\text { to cell are needed for efficient } \\
\text { replication } \\
\text { - Relatively low number of viral } \\
\text { progeny produced per cell } \\
\text { - Immunity to the virus is widely } \\
\text { present in the human population }\end{array}$ \\
\hline
\end{tabular}

One mutant has been approved for use in human head and neck cancer by the FDAequivalent of China

- Targeted mutants have been developed to receptors present in human tumor cells

Herpes simplex virus (OV)

Reovirus (OV)

Newcastle disease virus (OV)
- The function of all of its viral genes is known

- Safe in human brain

- Mutants that target p16/RB or MEK have been used

- High transgene capacity to allow for combination of OV-based and gene-therapy based therapy

- Rapid cell lysis

- Low number of viral particles per cell are required for efficient infection/replication

- Effective drugs are available to limit undesirable viral infection

- Safe in human brain

- Mutant strain targets cells that overexpress the oncogene ras

- High number of viral progeny produced per cell

- Function of all viral genes is known

- Safe when administered intravenously for human brain tumors

- Immunity not present in humans

- Strain of virus replicates in tumor cells due to their defective interferon response compared to normal cells

- High yield of viral progeny for infected cells
- Very immunogenic

- Immunity to the virus is widely present in human population

- Little experience in production for clinical trials

- Low interest by biotechnology community

- Function of many viral genes is still unknown

- Relatively low number of viral progeny produced per cell

- Immunity is widely present in the human population

- Experience and use for clinical trials confined to one biotechnology company

- RNA virus with high rate of mutation of viral genome

- Avian virus

- RNA virus with high rate of mutation of viral genome

- Little experience in production for clinical trials 
cule as the genome. After infection, the viral RNA genome is reverse transcribed into a double-stranded DNA, which can be integrated into the chromosomes of host cells and expressed as proteins. ${ }^{47,48} \mathrm{RV}$ s have been primarily used as vectors, although there are preclinical studies and a likely future attempt for a clinical trial using a retroviral-based OV. ${ }^{49}$ Recently, a replicationcompetent retroviral (RCR) vector based on the Moloney murine leukemia virus was found to replicate with kinetics similar to those of wild-type Moloney murine leukemia virus, and was also found to be stable through multiple serial passages in cultured cells. Injection of the RCR vector into established subcutaneous and intracranial tumors in mice resulted in highly efficient transmission of the transgene, and in some cases this resulted in transduction of the entire tumor. ${ }^{50}$ The following vector systems have been tested in preclinical studies RCRHSVtk/ganciclovir and RCR-CD/5-FC. One such RCR-CD vector is being developed for possible use in a clinical trial (Tocagen Inc.).

The advantages of RV vectors are that they are relatively easy to manipulate for gene therapy purposes and have been used widely. ${ }^{51}$ The available long-term experience with low toxicity to normal brain tissue makes this vector a safe candidate for CNS gene therapy. ${ }^{52,53}$ One of the problems of $\mathrm{RV}$ vectors is that the viral genome can be inserted randomly in the genome of the host. If the insertion happens to be in the middle of one of the host genes, this gene will be disrupted (insertional mutagenesis). If the disrupted host gene is involved in regulating cell division, uncontrolled cell division (i.e., cancer) can occur. ${ }^{54,55}$ Other disadvantages of RV vectors are low titers, instability of the viral particles, and small transgene capacity (the maximum amount of DNA that can be packaged into a retrovirus allows for only $7.5 \mathrm{~kb}$ of foreign DNA). Another drawback of RV vectors is the requirement that the target cell should be dividing for integration and expression of viral genes. This restricts gene therapy to proliferating cells only. ${ }^{56,57}$ Although $\mathrm{RV}$ vectors were used in the early gene therapy trials, their use has been greatly reduced in more recent gene therapy trials of cancer.

Adenoviral vectors. Adenoviruses (AV) are nonenveloped viruses containing a linear double-stranded DNA genome responsible for respiratory and eye infections in humans. For gene therapy purposes, the commonly used AV vectors are derived from a subgroup that can be manipulated to produce replication-deficient vectors. AVs are the most commonly used gene therapy vectors; in fact, they have become a common tool in the kit of the molecular biology lab whenever a gene needs to be expressed in a mammalian cell. The vector can be extensively modified to target it away from its usual receptor (present only in some cells ${ }^{58}$ ) to other receptors present to a desired target cell. ${ }^{59}$ Once bound to its receptor, the virus enters the cell in endosomal vesicles that fuse to lysosomes. ${ }^{60}$ Then the virus is able to liberate itself and escape to the cell nucleus for gene expression. Viral DNA does not generally integrate into the host genome and survives as an extrachromosomal element.

In terms of gene delivery to brain tumors, the vector has been used to deliver almost any type of anticancer gene. However, in clinical glioma trials, AV-mediated delivery of the genes for HSV $t k,{ }^{61} \mathrm{p} 53,{ }^{62}$ and human IF- $\beta^{63}$ have been published so far.

In summary, AV has been used widely as vectors in gene therapy trials, and the advantages to their use are relative ease of manipulation and ability to be produced at high titers. Furthermore, AV vectors are very efficient at transducing a wide variety of cells, both dividing and nondividing in vitro and in vivo. AVs do not usually integrate genetic material into the host genome, rather they replicate as episomal elements in the nucleus of the host cell with a transient gene expression, and consequently there is no risk of insertional mutagenesis. ${ }^{64}$ The limitations of AV vectors are their short-term gene expression and their production of toxic acute-phase responses. As clinical trials have begun to progress, it has become apparent that $\mathrm{AV}$ vectors may cause a significant innate immune response. ${ }^{65}$

Oncolytic adenovirus. Oncolytic AVs are engineered or naturally occurring strains of virus that replicate better in tumor cells versus normal cells. This selectivity can occur at three general levels. First, every time a virus infects a cell, an antiviral response occurs inside the cell consisting of numerous "stress" signals (FIG. 2). The overall effect of these signals is to limit the ability of the infecting virus to replicate and thus limit the number of viral progeny that could go on to infect neighboring cells. These "stress" signals consist of genes involved in the IF pathways, NF-kB, toll-like receptor responses, PKR, and others. ${ }^{66}$ In some cases, tumor cells have disabled some of these responses, because these responses tend to be pro-apoptotic and anti-proliferative. Therefore, tumor cells that have such disabled responses provide better targets for viral replication than normal cells with intact antiviral responses. Second, in tumor cells, several genes involved in cell-cycle regulation and apoptosis signaling are disrupted. These disruptions in tumor cell factors can be exploited to rationally engineer viral mutants that can not replicate well in normal cells with intact cell-cycle/apoptosis controls, but that will replicate in tumor cells that harbor these disruptions. Finally, viral mutants can be re-engineered so that they will target cell surface receptors present on tumor versus normal cells. For instance, an engineered oncolytic adenovirus (named ONYX-015) lacks a viral gene $(E 1 B)$ that encodes for a protein that inactivates the cellular tumor suppressor protein p53. This mutant AV was believed to replicate in and lyse p53-deficient human tumor 


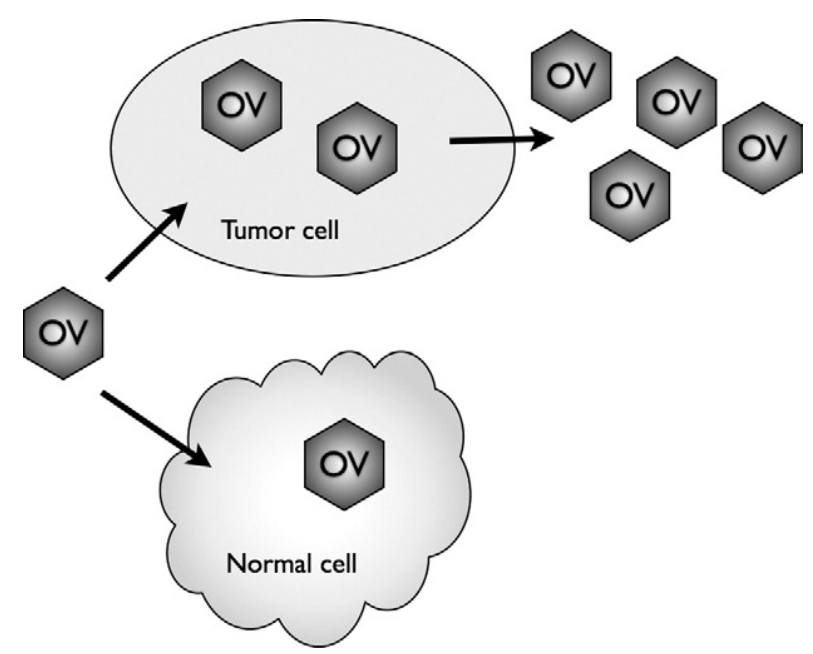

FIG. 2. General mechanism of OV selectivity. When most OVs (or any virus for that matter) infect a cell, a brisk antiviral ("danger" or "stress") response ensues that involves multiple intersecting and interconnected cellular pathways, the most prominent of which are the interferon pathways, the NF-kB pathway, the PKR (double stranded RNA-dependent protein kinase), and Toll-like receptor signaling pathways. The combined effect of these pathways is to shut off or limit viral replication (i.e., the production of viral progeny) so that neighboring cells do not become targets of such progeny. In tumor cells, some or all of these cellular mechanisms may be disrupted because of their pro-apoptotic and anti-proliferative effects. However, this allows the infecting virus to find an environment that is more propitious for replication, thus allowing for progeny viruses to be made and go on and infect neighboring cells.

cells, but not cells with functional p53. ${ }^{67,68}$ Based on gene mutations, this vector could be of use in as much as $50 \%$ of human GBMs carrying a p53 mutation. ${ }^{69}$ It may posses anticancer effects against the remaining tumors, because p53 function is modulated by $\mathrm{p} 14^{\mathrm{ARF}}$ protein with a gene $(C D K N 2 A)$ that is frequently deleted in GBM. $^{70,71}$ However, subsequent experiments with this virus have revealed that the mechanism of tumor selectivity is not based on the lack of p53 tumor suppressor function, rather on a more complicated mechanism related to the nuclear export of viral mRNAs in tumor cells. $^{72}$ Other oncolytic AVs include those that target defects in the $\mathrm{p} 16^{\mathrm{INK} 4 \mathrm{a}}$ tumor suppressor pathway. ${ }^{73}$ The p16 ${ }^{\mathrm{INK} 4 \mathrm{a}}$ protein shares the CDKN2A gene region with p $14^{\mathrm{ARF}}$ to make it one the most commonly mutated sides in gliomas.

HSV-1. HSV-1 is an enveloped neurotropic DNA virus. The wild type $\mathrm{HSV}-1$ is able to proceed into a lytic life cycle after infection or persist as an intranuclear episome in a latent state. Interest in the use of HSV-1 as a cancer attacking agent became high in the 1990s after reports showed that a genetically engineered form was oncolytic in brain tumor models. ${ }^{74}$ Since then, numerous reports have detailed various types of genetically engineered mutants for glioma therapy. The two most commonly used types have revolved around deletions of the viral genes (UL39 or UL40) that encode for the viral protein, ICP6. This protein possesses the function of a ribonucleotide reductase, and recent reports have linked this defect to an ability to replicate more selectively in cells with a defect in the p16 tumor suppressor gene. ${ }^{75}$ The other type has focused on a mutation of the viral gene that encodes for the viral protein ICP34.5. This viral gene counteracts a host cell response (based on the PKR enzyme) that promotes cellular apoptosis during infection. ${ }^{76}$ Mechanisms of tumor selectivity of this mutant virus have elicited several reports. In one case, authors have linked this viral defect to enhanced replication in tumor cells with elevated levels of Ras activity. ${ }^{77}$ However, others have disputed this claim and have linked mutant OV replication to activity of the MEK pathway present in the tumor more than normal cells. ${ }^{78,79}$ In addition, it is also possible that tumor cells may have lost some PKR function, thus enabling this mutant virus to replicate. ${ }^{80}$ Another genetically engineered HSV-1 (termed G207) was designed such that it is a replication competent in GBM, it has attenuated neurovirulence, it is temperature sensitive, and it can be eliminated with ganciclovir. ${ }^{81}$ It possesses deletion of the viral genes that encode both ICP6 and ICP34.5, and thus it may selectively target both cells with defects in p16 and with overexpression of MEK. Intraneoplastic inoculation with G207 decreased tumor growth and prolonged survival in animal models with no significant toxicity to the normal brain. Based on these results, two phase I studies using G207 in patients with anaplastic astrocytoma and GBM was completed with promising results. ${ }^{82-84}$

HSV-1 as an OV offers several advantages: 1) potential for incorporating a large load of foreign DNA; 2) infects human cells with high efficiency and low viral particle-to-cell ratio; 3) sensitivity to antiherpetic agents, such as ganciclovir or VCV providing a safety mechanism by which viral replication could be eliminated, if needed; and 4) the fact that HSV-1 never integrates into the host genome guarantees that the risk of insertional mutagenesis posed by $\mathrm{RV}$ vectors is not an issue with HSV-1. However, there are major challenges working with HSV-1. The genetic manipulation of HSV-1 is more difficult than that of AV due to the large size of the viral genome, and gene delivery could be affected due to pre-existing HSV immunity of the host (with as many as $90 \%$ of adults who have been exposed to HSV-1), and experience and interest in preclinical development by the biotechnology industry is limited. Moreover, the potential neurotoxicity of HSV-1 vectors could cause lifethreatening encephalitis from primary infection or from reactivation of latent virus.

Measles virus. The measles virus (MV) belongs to the Paramyxoviridae family, which are enveloped, single stranded RNA viruses. Numerous viruses are now being considered as potential cancer therapeutics, including Edmonston's vaccine strain of MV. The Edmonston vac- 
cine strain enters cells predominantly through the CD46 receptor, which interacts with cellular surface glycoproteins. ${ }^{85,86}$ The CD46 receptor is frequently overexpressed in tumors including gliomas, but it is expressed in low levels in the normal brain. ${ }^{87}$ In contrast to wild type virus, which can result in serious disease, vaccine strains of MV have an excellent safety record with millions of vaccine doses having been administered. Measles has been tested against a variety of cancer models, including gliomas, showing significant biologic effects. A phase I trial is currently being conducted using the MV in recurrent GBMs (E. Galanis, Mayo Clinic, personal communication).

Reovirus. Reovirus is a small RNA virus that produces self-limiting infections of the respiratory or intestinal tract, and it is endemic to the human population because most newborns get exposed to it. A strain of the virus was discovered to replicate in cells with an overactive Ras pathway, and this property has been exploited by attempting to define the safety and efficacy profile of this virus in clinical trials of humans with GBMs, as well as other cancers. ${ }^{88}$

As with most RNA viruses, reoviruses can replicate to very high levels in an infected cell, and thus in theory it could provide an increased therapeutic "punch," because the large number of viral progeny could infect a large number of neighboring tumor cells. Some of the issues that limit use of this virus relate to its small size and virological properties, which render genetic manipulation difficult. RNA viruses are also notoriously, highly mutable, which can also be problematic for production and use. The clinical experience is currently being supported by one biotechnology venture (Oncolytics Biotech Inc., Calgary, Alberta, Canada).

Newcastle disease virus (NDV). This virus (NDV) is an avian RNA virus. Several strains have been adapted for growth in tumor cells, and a phase I trial of intravenous infusion of an oncolytic NDV mutant in glioma patients has been reported. ${ }^{89}$ The advantages and disadvantages of this virus are similar to those of reovirus. However, differences with this virus exist; immunity to this avian virus does not exist in humans, and thus initial administration of the virus would not be limited by the adaptive arm of the host's immune reaction. This could also be a disadvantage, because the zoonotic nature of the virus and its administration into the human species could generate mutant species that become adapted to human infection and cause rapid pandemics, such as those that have occurred in the past with coronaviruses and HIV. The mechanism of selectivity of the virus for tumor cells is also not well understood. It may be due to the impaired IF and/or other host cell antiviral responses in tumor versus normal cells.

\section{GLIOMA GENE THERAPY TRIALS}

Six viruses (two replication-deficient and four replicating) have been studied in clinical brain tumor trials in which the results have been published (e.g., replicationdeficient retrovirus, replication-deficient adenovirus, replicating adenovirus, replicating HSV-1, replicating reovirus, and replicating NDV), and one replicating virus (measles) has been studied in glioma patients in a trial that is still ongoing and whose results have yet to be published.

$\mathrm{RV}$ and AV have been genetically modified to express HSV $t k$ with concurrent GCV administration. HSV and NDV have been used as an oncolytic agent. A selection of clinical trials is summarized in Table 2 . The first study using therapy in glioma patients used stereotactic intratumoral inoculation of an RV vector carrying $\mathrm{HSV} t k$ in 15 patients with recurrent malignant brain tumors. ${ }^{90} \mathrm{Al}-$ though this study showed some promising results in terms of antitumor efficacy, it was not a controlled or randomized protocol. Two subsequent phase I and II studies in patients with recurrent GBM were performed using an RV/HSV tk approach followed by GCV administration. The virus was delivered via vector-producing cells that were injected during tumor resection. The first study involved 12 patients and no treatment-related adverse effects were noted. There was an overall median survival of 6.8 months, with three patients surviving longer than 12 months and one patient who was recurrence-free 2.8 years after treatment. ${ }^{91}$ A comparable international, multicenter, uncontrolled study included 48 patients with recurrent gliomas. The median survival time was 8.6 months, with a 12-month survival rate of $27 \%$. Tumor recurrence was absent on MRI in seven patients for at least 6 months and in two patients for 12 months, and one patient remained recurrence-free at 24 months. ${ }^{92}$ A similar phase I study was performed in 12 children (aged 2 to 15 years) with recurrent malignant supratentorial tumors. This study also used an RV/HSV $t k /$ GCV approach. Disease progression occurred at a median time of 3 months after treatment, and the longest time until progression was 24 months with no adverse effects noted. ${ }^{93}$ A large controlled phase III trial seemed necessary for an ultimate confirmation of the efficacy of the RV/HSV $t k / G C V$ approach. This study used an adjuvant gene therapy protocol to the standard therapy of maximum surgical resection and irradiation for newly diagnosed GBM. After 4 years of follow-up of 248 patients who were divided into a gene therapy and a control arm, survival analysis showed no advantage of gene therapy in terms of tumor progression and overall survival. $^{94}$

There have been several clinical trials using AV vectors for malignant gliomas. Representative AV trials are briefly discussed herein. One clinical trial compared hu- 
Table 2. Selection of Closed Gene Therapy Trials in Malignant Glioma

\begin{tabular}{|c|c|c|c|c|}
\hline Phase & Strategy & No. of Patients & Median Survival (months) & Ref. \\
\hline & $\begin{array}{l}\text { Retrovirus VPC } \\
\text { HSV } t k / G C V\end{array}$ & & & \\
\hline I & Stereotactic injection & 15 & 8 & 90 \\
\hline I/II & Freehand injection & 48 & 9 & 91 \\
\hline III & Freehand injection & 248 (124 RV, 124 control) & $\begin{array}{l}12.2 \text { (RV) } \\
11.8 \text { (control) }\end{array}$ & 94 \\
\hline I & $\begin{array}{l}\text { Adenovirus vectors } \\
\text { Freehand injection } \\
\text { IFN } \beta\end{array}$ & 11 & 4 & 43 \\
\hline $\mathrm{II} / \mathrm{III}$ & $\begin{array}{l}\text { Freehand injection } \\
\text { HSVtk/GCV }\end{array}$ & 36 (17 AV, 19 control) & $\begin{array}{l}16 \text { (AV) } \\
9 \text { (control) }\end{array}$ & 96 \\
\hline I & $\begin{array}{l}\text { Oncolytic viruses } \\
\text { HSV-1 } \\
\text { Stereotactic injection }\end{array}$ & 9 & 9 & 97 \\
\hline I & $\begin{array}{l}\text { HSV-1 } \\
\text { Stereotactic injection }\end{array}$ & 21 & 6 & 83 \\
\hline I & $\begin{array}{l}\text { HSV-1 } \\
\text { Stereotactic injection }\end{array}$ & 6 & 6.6 & 84 \\
\hline I & $\begin{array}{l}\text { AV-p53 } \\
\text { AV (ONYX-015) }\end{array}$ & $\begin{array}{l}15 \\
24\end{array}$ & $\begin{array}{r}10 \\
6\end{array}$ & $\begin{array}{l}33 \\
103\end{array}$ \\
\hline
\end{tabular}

$\mathrm{AV}=$ adenovirus; $\mathrm{GCV}=$ ganciclovir; $\mathrm{HSV} t k=$ herpes simplex virus thymidine kinase; $\mathrm{RV}=$ retrovirus; $\mathrm{VPC}=$ vector producer cells.

mans with recurrent gliomas who were treated with direct stereotactic injection of either an RV vector or an AV vector that expressed the E. coli LacZ gene. ${ }^{95}$ They showed that the latter showed more widespread distribution of gene expression, and thus they concluded that AV vectors may be superior in their ability to distribute the transferred gene into brain tumors. A phase I/II trial evaluated an $\mathrm{AV}$ vector expressing the $\mathrm{HSV} t k$ gene in patients with primary or recurrent high-grade gliomas. This study was performed in a controlled randomized fashion on 36 patients (i.e., 17 in the treatment arm and 19 in the control group). All patients underwent surgical resection and AV injection, followed by intravenous GCV on postoperative day 5 for 14 days. The control group underwent resection without gene therapy and GCV injections. The median survival in the gene therapy group was significantly longer than the control group (62 vs. 37 weeks). ${ }^{96}$ As discussed previously, a large phase III trial has been conducted in Europe and Israel with this agent (Cerepro) and a recent statement from the company (Ark Therapeutics, Inc., London, United Kingdom) suggested evidence of efficacy, although published data would allow for independent evaluation of this claim. A recent phase I trial of AV-HSVtk delivered at the time of surgical resection of newly diagnosed malignant gliomas followed by oral VCV, combined with standard therapy (external field radiation to $6000 \mathrm{cGy}$ and temozolomide) has also recently concluded, showing relative safety, whereas the data is maturing regarding any evidence of efficacy (Chiocca et al., unpublished data). An AV vector delivering a wild-type copy of the p53 transgene was also evaluated in humans with recurrent gliomas. The vector was initially introduced by an implanted catheter placed in the middle of the tumor bed, followed by resection of the tumor to allow for studies related to p53 gene delivery and distribution. Again, while the treatment was well tolerated, the distribution of p53 gene into tumor was relatively low. ${ }^{33}$ One recent trial evaluated an $\mathrm{AV}$ vector in 11 patients with recurrent high-grade glioma introduced by stereotactic injection, followed by surgical resection and an additional injection of the vector into the tumor bed. ${ }^{43}$ The vector was well-tolerated in all but one patient who experienced confusion after the postoperative injection, which was believed to be caused by local brain toxicity. Tumor resection after the viral treatment demonstrated a dose-related inflammation and necrosis suggestive of a biologic effect from the transferred gene.

The next virus to undergo clinical trials in brain tumors was HSV-1. A phase I trial evaluated the safety of an oncolytic HSV-1 in nine patients with recurrent GBMs. Direct intratumoral injection was performed with no induction of encephalitis or adverse clinical symptoms, nor reactivation of latent HSV occurring, thus appearing as though tumor progression was controlled with some efficacy. ${ }^{97}$ Further investigations of the oncolytic HSV-1 mutant demonstrated that this vector is nontoxic when it is delivered into the tumor or into the adjacent brain. ${ }^{98}$ It was also shown that explanted glioma cells could still support replication of the oncolytic HSV-1, but that $20 \%$ of cells did not lyse immediately, but rather shedded low levels of virus for up to 31 days after which they lysed. ${ }^{99}$ A large phase III trial in Europe using HSV-1 is being conducted, which is sponsored by Crusade Laboratories (www.crusadelabs.com). Another clin- 
ical trial using a different HSV-1 mutant included 21 patients with recurrent glioma with similar results as they relate to safety. ${ }^{83}$

\section{CONCLUSIONS}

The rapid evolution of recombinant DNA technology enables us to develop new therapeutic modalities, including gene therapy. However, screening of new approaches is based on animal models that are far from representative of the analogous clinical scenarios, as shown in the discrepancy between the experimental animal studies and the clinical trials. As for brain tumors, the size, consistency, and extent of tumor models do not reflect the large, necrotic, infiltrative nature of GBMs. Approaches that aim at immune enhancement but use animal models that are poorly syngeneic to the implanted tumor are unlikely to reliably predict the human response. Accordingly, many ingenious gene therapy strategies effective in pre-clinical studies may not fulfill their expectations in the clinic. Furthermore, the problem of the delivery of genetic vectors into solid brain tumors and efficient in situ gene transfer remains one of the most significant hurdles in gene therapy. The efficiency of transduction could be improved by modifying vectorproducing cells, such as migratory vector-producing cells, which have the ability to track even single tumor cells invading the surrounding brain tissue. ${ }^{100,101}$ The currently used manual injection of vectors, which is limited in volume and can be injected at any given time, and the efflux that occurs along needle tracks, might be improved by the use of three-dimensional neuronavigation techniques and convection-enhanced delivery methods; these delivery methods involve the infusion of therapeutic agents via surgically implanted catheters, and they use a pressure gradient to achieve a greater volume of distribution compared with that evident with diffusion alone. $^{102}$

The completed brain tumor gene therapy trials have offered some promising results. However, the results of most clinical studies have not lived up to the expectations created by experimental data. Although gene delivery to human patients seems to be safe, these studies have not yet translated into benefits in the clinic. At present, gene therapy is being studied in trials for brain tumors, and so far it is not available outside of a clinical trial.

\section{REFERENCES}

1. Anwer K. Formulations for DNA delivery via electroporation in vivo. Methods Mol Biol 2008;423:77-89.

2. Rice J, Ottensmeier C, Stevenson F. DNA vaccines: precision tools for activating effective immunity against cancer. Nat Rev Cancer 2008;8:108-120.

3. Lechardeur D, Lukacs G. Nucleocytoplasmic transport of plasmid DNA: a perilous journey from the cytoplasm to the nucleus. Hum Gene Ther 2006;17:882-889.
4. Vaughan E, DeGiulio J, Dean D. Intracellular trafficking of plasmids for gene therapy: mechanisms of cytoplasmic movement and nuclear import. Curr Gene Ther 2006;6:671-681.

5. Bergen J, Park I, Horner P, Pun S. Nonviral approaches for neuronal delivery of nucleic acids. Pharm Res 2008;25:983-998.

6. Niidome T, Huang L. Gene therapy progress and prospects: nonviral vectors. Gene Ther 2002;9:1647-1652.

7. Faulds D, Heel R. Ganciclovir. A review of its antiviral activity, pharmacokinetic properties and therapeutic efficacy in cytomegalovirus infections. Drugs 1990;39:597-638.

8. Chen S, Shine H, Goodman J, Grossman R, Woo S. Gene therapy for brain tumors: regression of experimental gliomas by adenovirus-mediated gene transfer in vivo. Proc Natl Acad Sci U S A 1994;91:3054-3057.

9. Culver K, Ram Z, Wallbridge S, et al. In vivo gene transfer with retroviral vector-producer cells for treatment of experimental brain tumors. Science 1992;256:1550-1552.

10. Ezzeddine Z, Martuza R, Platika D, et al. Selective killing of glioma cells in culture and in vivo by retrovirus transfer of the herpes simplex virus thymidine kinase gene. New Biol 1991;3: $608-614$.

11. Ram Z, Culver K, Walbridge S, Blaese R, Oldfield E. In situ retroviral-mediated gene transfer for the treatment of brain tumors in rats. Cancer Res 1993;53:83-88.

12. Freeman S, Abboud C, Whartenby K, et al. The "bystander effect": tumor regression when a fraction of the tumor mass is genetically modified. Cancer Res 1993;53:5274-5283.

13. Fick J, Barker Fn, Dazin P, et al. The extent of heterocellular communication mediated by gap junctions is predictive of bystander tumor cytotoxicity in vitro. Proc Natl Acad Sci U S A 1995;92:11071-11075.

14. Ram Z, Walbridge S, Shawker T, et al. The effect of thymidine kinase transduction and ganciclovir therapy on tumor vasculature and growth of 9L gliomas in rats. J Neurosurg 1994;81:256-260.

15. Barba D, Hardin J, Sadelain M, Gage F. Development of antitumor immunity following thymidine kinase-mediated killing of experimental brain tumors. Proc Natl Acad Sci U S A 1994;91: $4348-4352$.

16. Kim J, Kim S, Kolozsvary A, et al. Selective enhancement of radiation response of herpes simplex virus thymidine kinase transduced 9L gliosarcoma cells in vitro and in vivo by antiviral agents. Int J Radiat Oncol Biol Phys 1995;33:861-868.

17. Nestler U, Wakimoto H, Siller-Lopez F, et al. The combination of adenoviral HSV TK gene therapy and radiation is effective in athymic mouse glioblastoma xenografts without increasing toxic side effects. J Neurooncol 2004;67:177-188.

18. Aghi M, Kramm C, Chou T, Breakefield X, Chiocca E. Synergistic anticancer effects of ganciclovir/thymidine kinase and 5 -fluorocytosine/cytosine deaminase gene therapies. J Natl Cancer Inst 1998;90:370-380.

19. Miller C, Williams C, Buchsbaum D, Gillespie G. Intratumoral 5-fluorouracil produced by cytosine deaminase/5-fluorocytosine gene therapy is effective for experimental human glioblastomas. Cancer Res 2002;62:773-780.

20. Wei M, Tamiya T, Chase M, et al. Experimental tumor therapy in mice using the cyclophosphamide-activating cytochrome P450 2B1 gene. Hum Gene Ther 1994;5:969-978.

21. Clarke L, Waxman D. Oxidative metabolism of cyclophosphamide: identification of the hepatic monooxygenase catalysts of drug activation. Cancer Res 1989;49:2344-2350.

22. Manome Y, Wen P, Chen L, et al. Gene therapy for malignan gliomas using replication incompetent retroviral and adenoviral vectors encoding the cytochrome P450 2B1 gene together with cyclophosphamide. Gene Ther 1996;3:513-520.

23. Wei M, Tamiya T, Rhee R, Breakefield X, Chiocca E. Diffusible cytotoxic metabolites contribute to the in vitro bystander effect associated with the cyclophosphamide/cytochrome P450 2B1 cancer gene therapy paradigm. Clin Cancer Res 1995;1:11711177.

24. Sathornsumetee S, Reardon D, Desjardins A, et al. Molecularly targeted therapy for malignant glioma. Cancer 2007;110:13-24.

25. Vousden K, Lane D. p53 in health and disease. Nat Rev Mol Cell Biol 2007;8:275-283. 
26. Louis D, Holland E, Cairncross J. Glioma classification: a molecular reappraisal. Am J Pathol 2001;159:779-786.

27. Li H, Alonso-Vanegas M, Colicos M, et al. Intracerebral adenovirus-mediated p53 tumor suppressor gene therapy for experimental human glioma. Clin Cancer Res 1999;5:637-642.

28. Roth J. Adenovirus p53 gene therapy. Expert Opin Biol Ther 2006;6:55-61.

29. von Deimling A, Louis D, Wiestler O. Molecular pathways in the formation of gliomas. Glia 1995;15:328-338.

30. Louis D. Molecular pathology of malignant gliomas. Annu Rev Pathol 2006;1:97-117.

31. Inoue $\mathrm{R}$, Moghaddam $\mathrm{K}$, Ranasinghe $\mathrm{M}$, et al. Infectious delivery of the $132 \mathrm{~kb}$ CDKN2A/CDKN2B genomic DNA region results in correctly spliced gene expression and growth suppression in glioma cells. Gene Ther 2004;11:1195-1204.

32. Huang P, Cavenee W, Furnari F, White F. Uncovering therapeutic targets for glioblastoma: a systems biology approach. Cell Cycle 2007;6:2750-2754.

33. Lang F, Bruner J, Fuller G, et al. Phase I trial of adenovirusmediated p53 gene therapy for recurrent glioma: biological and clinical results. J Clin Oncol 2003;21:2508-2518.

34. Gansbacher B, Zier K, Daniels B, et al. Interleukin 2 gene transfer into tumor cells abrogates tumorigenicity and induces protective immunity. J Exp Med 1990;172:1217-1224.

35. Sobol R, Fakhrai H, Shawler D, et al. Interleukin-2 gene therapy in a patient with glioblastoma. Gene Ther 1995;2:164-167.

36. Yu J, Wei M, Chiocca E, Martuza R, Tepper R. Treatment of glioma by engineered interleukin 4 -secreting cells. Cancer Res 1993;53:3125-3128.

37. Parker J, Gillespie G, Love C, et al. Engineered herpes simplex virus expressing IL-12 in the treatment of experimental murine brain tumors. Proc Natl Acad Sci U S A 2000;97:2208-2213.

38. Qin X, Tao N, Dergay A, et al. Interferon-beta gene therapy inhibits tumor formation and causes regression of established tumors in immune-deficient mice. Proc Natl Acad Sci U S A 1998;95:14411-14416.

39. Gansbacher B, Bannerji R, Daniels B, et al. Retroviral vectormediated gamma-interferon gene transfer into tumor cells generates potent and long lasting antitumor immunity. Cancer Res 1990;50:7820-7825.

40. Herrlinger U, Kramm C, Johnston K, et al. Vaccination for experimental gliomas using GM-CSF-transduced glioma cells. Cancer Gene Ther 1997;4:345-352.

41. Fearon E, Pardoll D, Itaya T, et al. Interleukin-2 production by tumor cells bypasses $\mathrm{T}$ helper function in the generation of an antitumor response. Cell 1990;60:397-403.

42. Tjuvajev J, Gansbacher B, Desai R, et al. RG-2 glioma growth attenuation and severe brain edema caused by local production of interleukin-2 and interferon-gamma. Cancer Res 1995;55:19021910.

43. Chiocca E, Smith K, McKinney B, et al. A phase I trial of Ad.hIFN-beta gene therapy for glioma. Mol Ther 2008;16:618626.

44. Im S, Gomez-Manzano C, Fueyo J, et al. Antiangiogenesis treatment for gliomas: transfer of antisense-vascular endothelial growth factor inhibits tumor growth in vivo. Cancer Res 1999; 59:895-900.

45. Millauer B, Shawver L, Plate K, Risau W, Ullrich A. Glioblastoma growth inhibited in vivo by a dominant-negative Flk-1 mutant. Nature 1994;367:576-579.

46. Van Meir E, Polverini P, Chazin V, et al. Release of an inhibitor of angiogenesis upon induction of wild type p53 expression in glioblastoma cells. Nat Genet 1994;8:171-176.

47. Kim S, Kim S, Robbins P. Retroviral vectors. Adv Virus Res 2000;55:545-563.

48. Young L, Searle P, Onion D, Mautner V. Viral gene therapy strategies: from basic science to clinical application. J Pathol 2006;208:299-318.

49. Tai C, Kasahara N. Replication-competent retrovirus vectors for cancer gene therapy. Front Biosci 2008;13:3083-3095.

50. Wang W, Tai CK, Kershaw AD, et al. Use of replication-competent retroviral vectors in an immunocompetent intracranial glioma model. Neurosurg Focus 2006;20:E25.
51. Rainov N, Kramm C. Recombinant retrovirus vectors for treatment of malignant brain tumors. Int Rev Neurobiol 2003;55:185203.

52. Ram Z, Culver K, Walbridge S, et al. Toxicity studies of retroviral-mediated gene transfer for the treatment of brain tumors. J Neurosurg 1993;79:400-407.

53. Long Z, Li L, Grooms T, et al. Biosafety monitoring of patients receiving intracerebral injections of murine retroviral vector producer cells. Hum Gene Ther 1998;9:1165-1172.

54. Uren A, Kool J, Berns A, van Lohuizen M. Retroviral insertional mutagenesis: past, present and future. Oncogene 2005;24:76567672 .

55. Fehse B, Roeder I. Insertional mutagenesis and clonal dominance: biological and statistical considerations. Gene Ther 2008;15:143153.

56. Roth J, Nguyen D, Lawrence D, et al. Retrovirus-mediated wildtype p53 gene transfer to tumors of patients with lung cancer. Nat Med 1996;2:985-991.

57. Tait D, Obermiller P, Redlin-Frazier S, et al. A phase I trial of retroviral BRCA1sv gene therapy in ovarian cancer. Clin Cancer Res 1997;3:1959-1968.

58. Freimuth P, Philipson L, Carson S. The coxsackievirus and adenovirus receptor. Curr Top Microbiol Immunol 2008;323:67-87.

59. Waehler R, Russell S, Curiel D. Engineering targeted viral vectors for gene therapy. Nat Rev Genet 2007;8:573-587.

60. Douglas J. Adenoviral vectors for gene therapy. Mol Biotechnol 2007;36:71-80.

61. Sandmair A, Vapalahti M, Yla-Herttuala S. Adenovirus-mediated herpes simplex thymidine kinase gene therapy for brain tumors. Adv Exp Med Biol 2000;465:163-170.

62. Vecil G, Lang F. Clinical trials of adenoviruses in brain tumors: a review of Ad-p53 and oncolytic adenoviruses. J Neurooncol 2003;65:237-246.

63. Chiocca EA, Smith KM, McKinney B, et al. A phase I trial of Ad.hIFN-beta gene therapy for glioma. Mol Ther 2008;16:618626.

64. Verma I, Somia N. Gene therapy — promises, problems and prospects. Nature 1997;389:239-242.

65. Hartman Z, Appledorn D, Amalfitano A. Adenovirus vector induced innate immune responses: impact upon efficacy and toxicity in gene therapy and vaccine applications. Virus Res 2008;132: $1-14$.

66. Chiocca E. The host response to cancer virotherapy. Curr Opin Mol Ther 2008;10:38-45.

67. Bischoff J, Kirn D, Williams A, et al. An adenovirus mutant that replicates selectively in p53-deficient human tumor cells. Science 1996;274:373-376.

68. Heise C, Sampson-Johannes A, Williams A, et al. ONYX-015, an E1B gene-attenuated adenovirus, causes tumor-specific cytolysis and antitumoral efficacy that can be augmented by standard chemotherapeutic agents. Nat Med 1997;3:639-645.

69. Hollstein M, Rice K, Greenblatt M, et al. Database of p53 gene somatic mutations in human tumors and cell lines. Nucleic Acids Res 1994;22:3551-3555.

70. Rutka J, Akiyama Y, Lee S, et al. Alterations of the p53 and pRB pathways in human astrocytoma. Brain Tumor Pathol 2000;17: 65-70.

71. Sherr C. The INK4a/ARF network in tumour suppression. Nat Rev Mol Cell Biol 2001;2:731-737.

72. O'Shea C, Johnson L, Bagus B, et al. Late viral RNA export, rather than p53 inactivation, determines ONYX-015 tumor selectivity. Cancer Cell 2004;6:611-623.

73. Heise C, Hermiston T, Johnson L, et al. An adenovirus E1A mutant that demonstrates potent and selective systemic anti-tumoral efficacy. Nat Med 2000;6:1134-1139.

74. Martuza R, Malick A, Markert J, Ruffner K, Coen D. Experimental therapy of human glioma by means of a genetically engineered virus mutant. Science 1991;252:854-856.

75. Aghi M, Visted T, Depinho R, Chiocca E. Oncolytic herpes virus with defective ICP6 specifically replicates in quiescent cells with homozygous genetic mutations in p16. Oncogene 2008;27:42494254. 
76. Cassady K, Gross M, Roizman B. The second-site mutation in the herpes simplex virus recombinants lacking the gamma134.5 genes precludes shutoff of protein synthesis by blocking the phosphorylation of eIF-2alpha. J Virol 1998;72:7005-7011.

77. Farassati F, Yang A, Lee P. Oncogenes in Ras signalling pathway dictate host-cell permissiveness to herpes simplex virus 1. Nat Cell Biol 2001;3:745-750.

78. Smith K, Mezhir J, Bickenbach K, et al. Activated MEK suppresses activation of PKR and enables efficient replication and in vivo oncolysis by Deltagamma(1)34.5 mutants of herpes simplex virus 1. J Virol 2006;80:1110-1120.

79. Veerapong J, Bickenbach K, Shao M, et al. Systemic delivery of (gamma1)34.5-deleted herpes simplex virus-1 selectively targets and treats distant human xenograft tumors that express high MEK activity. Cancer Res 2007;67:8301-8306.

80. Balachandran S, Barber G. PKR in innate immunity, cancer, and viral oncolysis. Methods Mol Biol 2007;383:277-301.

81. Mineta T, Rabkin S, Yazaki T, Hunter W, Martuza R. Attenuated multi-mutated herpes simplex virus- 1 for the treatment of malignant gliomas. Nat Med 1995;1:938-943.

82. Aghi MK, Chiocca EA. Phase Ib trial of oncolytic herpes virus G207 shows safety of multiple injections and documents viral replication. Mol Ther 2009;17:8-9.

83. Markert J, Medlock M, Rabkin S, et al. Conditionally replicating herpes simplex virus mutant, G207 for the treatment of malignant glioma: results of a phase I trial. Gene Ther 2000;7:867-874.

84. Markert JM, Liechty PG, Wang W, et al. Phase Ib trial of mutant herpes simplex virus G207 inoculated pre-and post-tumor resection for recurrent GBM. Mol Ther 2009;17:199-207.

85. Dorig RE, Marcil A, Chopra A, Richardson CD. The human CD46 molecule is a receptor for measles virus (Edmonston strain). Cell 1993;75:295-305.

86. Schneider U, von Messling V, Devaux P, Cattaneo R. Efficiency of measles virus entry and dissemination through different receptors. J Virol 2002;76:7460-7467.

87. Allen C, Paraskevakou G, Liu C, et al. Oncolytic measles virus strains in the treatment of gliomas. Expert Opin Biol Ther 2008; 8:213-220.

88. Forsyth P, Roldan G, George D, et al. A phase I trial of intratumoral administration of reovirus in patients with histologically confirmed recurrent malignant gliomas. Mol Ther 2008;16:627632.

89. Freeman A, Zakay-Rones Z, Gomori J, et al. Phase I/II trial of intravenous NDV-HUJ oncolytic virus in recurrent glioblastoma multiforme. Mol Ther 2006;13:221-228.

90. Ram Z, Culver K, Oshiro E, et al. Therapy of malignant brain tumors by intratumoral implantation of retroviral vector-producing cells. Nat Med 1997;3:1354-1361.
91. Klatzmann D, Valery C, Bensimon G, et al. A phase I/II study of herpes simplex virus type 1 thymidine kinase "suicide" gene therapy for recurrent glioblastoma. Study Group on Gene Therapy for Glioblastoma. Hum Gene Ther 1998;9:2595-2604.

92. Shand N, Weber F, Mariani L, et al. A phase 1-2 clinical trial of gene therapy for recurrent glioblastoma multiforme by tumor transduction with the herpes simplex thymidine kinase gene followed by ganciclovir. GLI328 European-Canadian Study Group. Hum Gene Ther 1999;10:2325-2335.

93. Packer R, Raffel C, Villablanca J, et al. Treatment of progressive or recurrent pediatric malignant supratentorial brain tumors with herpes simplex virus thymidine kinase gene vector-producer cells followed by intravenous ganciclovir administration. J Neurosurg 2000;92:249-254.

94. Rainov N. A phase III clinical evaluation of herpes simplex virus type 1 thymidine kinase and ganciclovir gene therapy as an adjuvant to surgical resection and radiation in adults with previously untreated glioblastoma multiforme. Hum Gene Ther 2000;11: 2389-2401.

95. Puumalainen A, Vapalahti M, Agrawal R, et al. Beta-galactosidase gene transfer to human malignant glioma in vivo using replication-deficient retroviruses and adenoviruses. Hum Gene Ther 1998;9:1769-1774.

96. Immonen A, Vapalahti M, Tyynela K, et al. AdvHSV-tk gene therapy with intravenous ganciclovir improves survival in human malignant glioma: a randomized, controlled study. Mol Ther 2004;10:967-972.

97. Rampling R, Cruickshank G, Papanastassiou V, et al. Toxicity evaluation of replication-competent herpes simplex virus (ICP 34.5 null mutant 1716 ) in patients with recurrent malignant glioma. Gene Ther 2000;7:859-866.

98. Harrow S, Papanastassiou V, Harland J, et al. HSV1716 injection into the brain adjacent to tumour following surgical resection of high-grade glioma: safety data and long-term survival. Gene Ther 2004; $11: 1648-1658$.

99. Harland J, Papanastassiou V, Brown SM. HSV1716 persistence in primary human glioma cells in vitro. Gene Ther 2002;9:11941198.

100. Herrlinger U, Woiciechowski C, Sena-Esteves M, et al. Neural precursor cells for delivery of replication-conditional HSV-1 vectors to intracerebral gliomas. Mol Ther 2000;1:347-357.

101. Aboody KS, Brown A, Rainov NG, et al. Neural stem cells display extensive tropism for pathology in adult brain: evidence from intracranial gliomas. Proc Natl Acad Sci U S A 2000;97: 12846-12851.

102. Ferguson SD, Foster K, Yamini B. Convection-enhanced delivery for treatment of brain tumors. Expert Rev Anticancer Ther 2007; 7:S79-85. 